\title{
Investigating the influence of the core material on the mechanical performance of a nitinol wire wrapped helical auxetic yarn.
}

FAISAL, N.H., FOWLIE, A., CONNELL, J., MACKENZIE, S., NOBLE, R. and PRATHURU, A.K. 


\title{
Investigating the influence of the core material on the mechanical performance of a nitinol wire wrapped helical auxetic yarn
}

\author{
Nadimul Haque Faisal $\mathbb{D}$, Andrew Fowlie, Joe Connell, Sean Mackenzie, \\ Ryan Noble and Anil Kumar Prathuru
}

\begin{abstract}
Helical Auxetic Yarns (HAYs) can be used in a variety of applications from healthcare to blast and impact resistance. This work focuses on the effect of the use of different core materials (e.g. rubber, polyurethane, polytetrafluoroethylene/ teflon, polypropylene, polyetheretherketone, polycarbonate, acetal) with a nitinol wire wrap component on the maximum Negative Poisson Ratio (NPR) produced and thus the auxetic performance of Helical Auxetic Yarns (HAYs). From the analytical model, it was found that an acetal core produced the largest NPR when compared to the other six materials. The trend obtained from the experimental tensile tests (validation) correlated closely with the theoretical predictions of the NPR as axial strain was increased. The experimental method presented a maximum NPR at an average axial strain of 0.148 which was close to the strain of 0.155 predicted by theory. However, the maximum experimental NPR was significantly lower than that predicted by the analytical model.
\end{abstract}

\section{Keywords}

Helical auxetic yarn, design parameters, negative Poisson's ratio, nitinol, mechanical performance, safety applications

Date received: 17 January 202I; accepted: 3 June 2021

\section{Introduction}

Helical auxetic yarn (HAY) can be woven into a textilelike 3D structure capable of resisting greater loads than standard materials which has led to suggestions that they could be used to reinforce and protect existing critical structures such as pressure vessels or the exterior of an aeroplane. Auxetics composites are already in use for many applications where their exclusive properties are beneficial. However, the problem is that the study of HAY's is a relatively new field and not a great deal of work has been done to optimise designs and investigate potential component materials. If it were possible to optimise the design of a single HAY, then it would suggest that stronger, more effective $2 \mathrm{D}$ or $3 \mathrm{D}$ structures can be constructed. It would then be possible to manipulate the negative Poisson's ratio, (NPR), of the structure by varying the geometry and mechanical properties of the individual HAYs.

Wright et al. ${ }^{1}$ investigated the effects of wrap angle on the performance of three different of HAYs subjected to a $20 \%$ strain to be used as bandages in health applications. It was established from the test that when the load was initially applied, there was little strain experienced by the HAY. However, as the load increased, the helically wound wrap began to straighten and the strain rapidly increased. Different wrap angles had the same general trend, but the onset of high strain values changed significantly. The study also highlighted the significance of good quality control and the importance of producing comparable test specimens. Results from the HAYs they manufactured had a standard deviation of just $4 \%$, meaning that the manufacturing process was well controlled. However, small geometrical differences in the HAYs, lead to large differences in their behaviour whilst under loading.

Bhattacharya et al. ${ }^{2}$ investigated the physical interaction between the core and wrap components of the HAY and the effect that such interactions had on the

School of Engineering, Robert Gordon University, Aberdeen, UK

\section{Corresponding author:}

Nadimul Haque Faisal, School of Engineering, Robert Gordon University, Garthdee Road, Aberdeen, ABI0 7GJ, UK.

Email: N.H.Faisal@rgu.ac.uk 
auxetic behaviour. They studied the relationship between the indentation on the core caused by the wrap and the relative Young's moduli of both components. This was achieved by manufacturing HAYs with the following core and wrap materials: core (Polyurethane (PU), Polyamide 12 (PA), Polyethylene (PE), Polyethylene terephthalate (PET)) and wrap (Ultrahigh-molecular-weight polyethylene (UHMWPE), Copolymer polyamide (CPPA)).

A low initial wrap angle of $12^{\circ}$ was used for each HAY, as from their previous work this was found to provide the best auxetic behaviour. To investigate the effect of indentation, cross sectional images of the HAY were taken under a microscope after tensile testing. They observed a large difference in component moduli lead to a decrease in the auxetic effect and therefore concluded that there must be an adequate difference between the Young's modulus of both wrap and core to produce desirable auxetic effects, but it must also be low enough to prevent indentation of the wrap into the core from occurring. Balancing these conditions will lead to a HAY with an optimum NPR.

A study by Zhang et al. ${ }^{3}$ suggested an improved design by enclosing the HAY within a sheath coating. This idea was suggested to try and overcome problems such as slippage between fibres that make the pitch angle uncontrollable. The sheath could also act as a protective layer allowing for more delicate configurations to be used as well as increased defence against corrosion and wear. The yarn was made from a silicone rubber core and a polyethylene wrap while the sheath was made from a silicone gel. An experimental study was then conducted on this new design and focused on the mechanical performance. The thickness of coating was increased three times, and the effect of this on the HAY's performance was graphed to determine the Poisson's ratio. Results showed that as the coating became thicker, the auxetic effect was impeded. It was concluded that a coating should only be used when it is necessary to provide additional corrosion and wear resistance. Du et al. ${ }^{4}$ followed up their theoretical study ${ }^{5}$ to verify their analytical model with an experiment. A HAY with a rubber core and nylon wrap was produced using a ring-spinning system. Experimental results using these materials showed radial expansion of the HAY when under a tensile load thus producing an NPR. Furthermore, this expansion was seen to be greater in HAYs with higher diametric ratios of the core to the wrap, lower wrap angles and higher Young's modulus wraps. Experimental results showed a strong correlation with those from theory.

Zhang et al. ${ }^{6}$ studied the effects of the auxetic behaviour of HAYs as the component modulus, core/wrap diameter ratio and initial wrap angle were varied. HAYs were constructed using an Elastollan ${ }^{\circledR}$ thermoplastic polyurethane (TPU) core and due to their high strength and modulus, ultra-high molecular-weight polyethylene (UHMWPE) and stainless steel were each used for the wrap materials. It was found that a large difference in the Young's modulus of the core and wrap yielded better auxetic performance. Furthermore, they found that a higher core/wrap diameter ratio and lower initial wrap angle produced a greater maximum NPR. Further findings identified that the instantaneous Poisson's ratio could not be accurately predicted for this highly non-linear material from the ratio of transverse to axial strain. This inaccuracy in the prediction of the Poisson's ratio was seen to increase at lower wrap angles. Following on from their previous work, they also investigated the dynamic thermo-mechanical and impact properties of HAYs (Zhang et al. ${ }^{6}$ ). This was done by fabricating HAYs with thermoplastic polyurethane core and UHMWPE or stainless-steel wrap and performing dynamic mechanical analysis (DMA). From the results of the DMA, it was established that the core/wrap diameter ratio and the initial wrap angle heavily influenced the dynamic thermomechanical behaviour of HAYs. Results from a highrate tensile impact test showed that fibre property, impact velocity and initial wrap angle had a great effect on the impact response of HAY. They also determined that for their configuration of HAY, an initial wrap angle of $27^{\circ}$ was found to give the best combination of stiffness, energy absorption and auxetic performance of HAYs. This disagrees with what Bhattacharya et al. ${ }^{2}$ presented regarding an optimum initial wrap angle of $12^{\circ}$. This suggests that the optimum wrap angle varies significantly when varying other parameters.

In some of the recent exemplary work, for example, Nazir et al. ${ }^{7}$ developed HAY for filtration or energy absorbing applications by wrapping a stiffer yarn (i.e. multifilament Kevlar) on core yarn (i.e. multifilament Polypropylene), and obtained maximum auxetic effect by varying wrap to core angle at $8^{\circ}$. Similarly, Ullah et al., ${ }^{8}$ developed HAY by using a combination of highperformance fibres and conventional fibres and studied the effect of different levels of twist per metre. It was observed that the core filament of HAY increased its thickness in transverse direction under stress with considerable NPR, and its values indicated that the auxeticity had a direct relation with core filament thickness or diameter and inversely proportional to the linear density of wrap filament, in case of the wrap angle the auxeticity of HAY yarn had an inverse effect with wrap angle. Material wise, Kevlar/polypropylene combination showed maximum auxeticity at $15^{\circ}$ wrap angle while Kevlar/nylon combination sample showed minimum auxeticity at $25^{\circ}$ wrap angle.

In this study, we aim to investigate the influence of the use of different core materials (i.e. rubber, polyurethane, polytetrafluoroethylene (Teflon), polypropylene, polyetheretherketone, polycarbonate, acetal) on the mechanical performance, and thus the auxetic performance, of a nitinol wrapped helical auxetic yarn.

\section{Analytical methodology}

To validate and compare results, analytical and experimental methods were used. The analytical model 


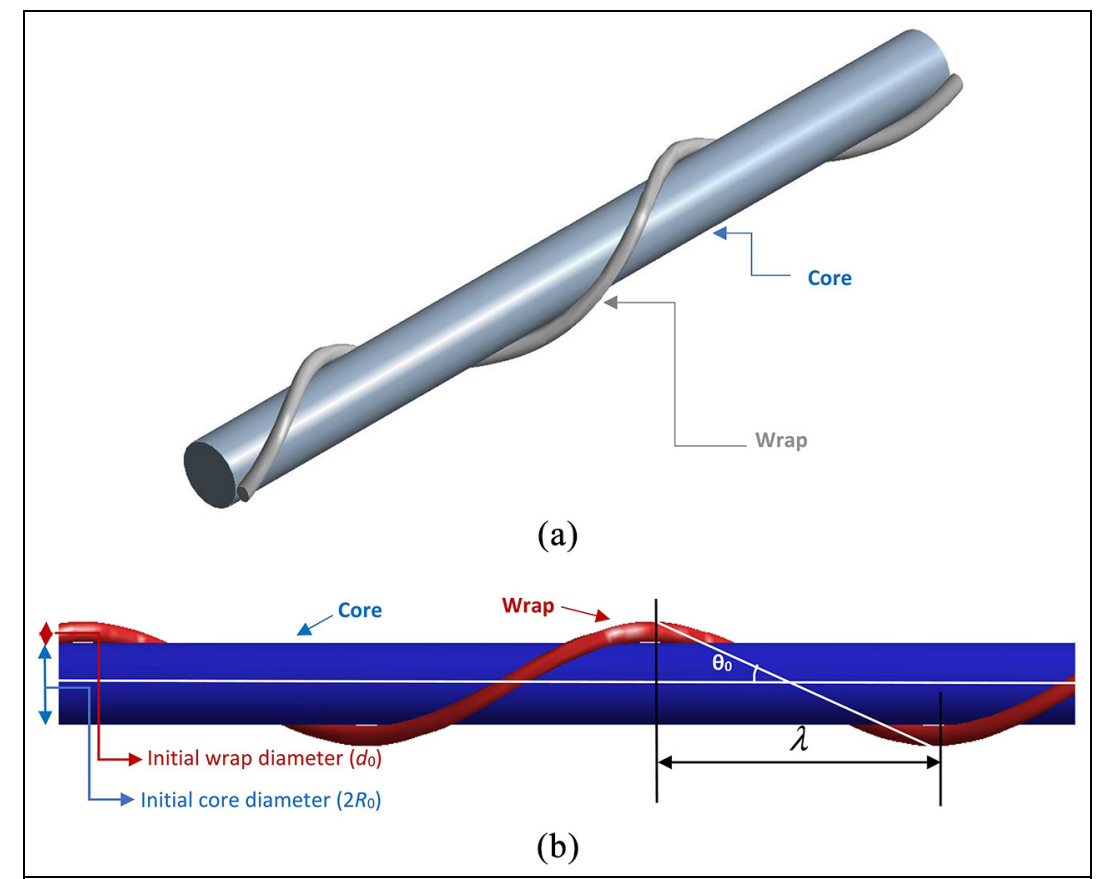

Figure I. HAY model showing Stage I of deformation (straight core with helically wound wrap): (a) two cycle model created in SOLIDWORKS ${ }^{\circledR} 3 D$ CAD software (isometric view) with wrap angle of $30^{\circ}$ and (b) connections with initial wrap angle, $\theta_{0}$, and initial helical pitch, $\lambda$.

proposed by $\mathrm{Du}$ et al. $^{5}$ was recreated using Mathcad $^{\circledR} 15$ for this investigation because it provided the most streamlined approach to changing the parameters that were under investigation. For the experiment, a tensile test was performed on a HAY sample to validate the results of maximum NPR calculated by theory. This enabled the maximum NPR of further material combinations to be proposed with reliable backing.

Assumptions made by the analytical model are: (1) the Poisson's ratio and Young's modulus of the wrap and core remain constant when in tension, (2) the wrap fibre experiences no elongation before it is pulled straight and lies in the centre of the HAY, and (3) the tensile process is divided into two stages: (a) Stage $1-$ the wrap fibre changes from being wound around the core and unravels, displacing the core, as tension is applied until it is pulled straight and lies in the centre of the HAY (Figure 1), and (b) Stage 2 - the wrap filament remains in the centre of the HAY but now experiences elongation and deforms radially with the core fibre for the remainder of the tensile process. While providing a full description of analytical approach is out-with the scope of this paper, a good description to the topic can be found in Du et al., ${ }^{5}$ however, key solution steps has been provided in Supplemental Appendix A (refer Supplemental Material).

With effective diameter during the second stage $(D)$ derived (equation (A.21)) (refer Appendix A: Supplemental Material), the Poisson's ratio ( $\nu$ ) of the yarn can be derived by manipulating the standard equation: $\nu=-\frac{\varepsilon_{\text {transverse }}}{\varepsilon_{\text {axial }}}$ to become $\nu=-\frac{\frac{D-2\left(R_{0}+d_{0}\right)}{2\left(R_{0}+d_{0}\right)}}{\varepsilon_{\text {crit }}}$,

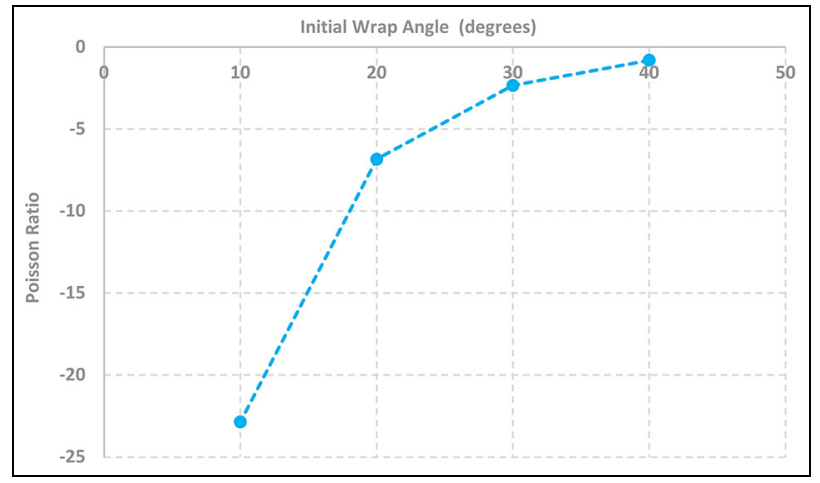

Figure 2. Maximum negative instantaneous Poisson's ratio when varying initial wrap angle.

where $\varepsilon_{\text {crit }}=\left(\frac{1}{\cos \theta_{0}}-1\right)$, and $R_{0}$ and $d_{0}$ remain the initial core radius and initial wrap diameter, respectively.

Initial findings from the analytical model found that the maximum negative instantaneous Poisson's ratio achievable by any given HAY would tend to infinity as the initial wrap angle approaches zero. The graph (Figure 2) was plotted by going through the analytical model for four different initial wrap angles $\left(\theta_{0}\right)$. For each wrap angle, a new critical strain was calculated using the equations shown above. From experimental studies (e.g. Zhang et al. ${ }^{6}$ and Zhang et al. ${ }^{9}$ ) it was clear that the maximum NPR predicted by analytical model greatly exaggerated what was possible to achieve in an experimental setting. These findings can be seen in Figure 2, and closely represent the findings of Zhang et al. ${ }^{6}$ and Zhang et al., ${ }^{9}$ where it was found that the 
maximum NPR increases exponentially as the initial wrap angle is smaller. It also showed the uncertainty in the true Poisson's ratio (i.e. it increases significantly from $30^{\circ}$ to $20^{\circ}$ and even more so from $20^{\circ}$ to $10^{\circ}$, and therefore, to achieve reliable results from this model an initial wrap angle of $30^{\circ}$ was opted, as shown in isometric view in Figure 1(a).

\section{HAY component material selection}

Helical auxetic yarn type structures are fabricated by using two or more different materials to develop a new composite material that can have superior mechanical properties than its constituents, when used alone. If the selection of the constituent materials is strategically done (considering industrial sectors such as defence, safety, healthcare, oil and gas, aerospace and fashion for range of structural application) then such HAY structure could also offer range of advantages, such as desirable combination of weight, strength, stiffness and toughness.

Among many, a material that could enhance the mechanical performance of a HAY is a nickel titanium (Ni-Ti) alloy commonly known as 'nitinol' which is superplastic. Superplastic behaviour is a property where plastic effects are suppressed, and the subject can return to its original form after undergoing significant deformation. ${ }^{10}$ It is primarily used in the medical industry for braces and guidewires due to the metal having a high tensile strength of between 754 and $900 \mathrm{MPa}$ and because it can retain its original form. Nitinol can also withstand greater strains (i.e. large deformation) prior to failure compared to traditional metal alloys, enduring cyclic strain amplitudes ranging from $4 \%$ to $12 \%{ }^{11}$ Nitinol can be a material of choice for applications requiring significant flexibility during dynamic loading. These desirable properties along with corrosion resistance, wear resistance properties, and ability to sense cracks and contract to heal macro-cracks are attributes that could be beneficial if used as the wrap in a HAY structure.

Rubber has the advantage of being cheap compared to other polymers so could be used to create a costeffective HAY. For example, a carpet like structure could be constructed and used to blanket underwater pipelines to mitigate damage from marine debris and erosion. Applications in extreme cold environments should be overlooked because rubber becomes brittle at low temperatures.

Study found that auxetic polyurethane foams demonstrated an increase in stiffness due to compressive loads being applied, which leads to a decrease in the magnitude of the compressive stresses being applied to the polyurethane foam. This foam could therefore be used in the HAY's core to create a fabric suited to protective gloves for workers exposed to severe vibrations produced by heavy machinery. ${ }^{12}$ This could also be used for other vibration dampening applications such as sound proofing or blast curtains.
Polytetrafluoroethylene (PTFE), commonly known as Teflon ${ }^{\mathrm{TM}}$, used in HAYs could provide an increase in auxetic performance due to PTFE having a very low friction coefficient when rubbing against metallic surfaces. ${ }^{13}$ The lack of friction would potentially extend the useable life of a HAY due to less abrasion between the HAY components. An extension in useable life would be particularly useful in applications such as medicine where replacement/maintenance is difficult, and failures can be catastrophic. In the biomedical field, auxetics are used to open arteries or cavities within the body. While under tension, PTFE will expand laterally and provide the force necessary to open any closed or collapsed cavities. ${ }^{14,15}$

Alderson et al. ${ }^{16}$ found that auxetic polypropylene (PP) fibres could be used in high load applications because auxetic PP fibres could carry twice the maximum load of conventional PP fibres when being used as a reinforcement in composites. As the composites were loaded, the auxetic PP expanded laterally when tightened, resulting in a self-locking mechanism with a softened epoxy resin.

Advanced Fabric Technologies manufacture several auxetic composite materials called Xtegra for use in applications where impact resistance is the primary consideration. For example, blast curtains used in the oil and gas industry to protect critical assets from impacts. Furthermore, these high impact resistant materials are perfectly suited in many military and defence applications such as, blast and ballistic protection, body armour, mine safety and jet engine shielding. ${ }^{17}$ Not melting until $343^{\circ} \mathrm{C}^{18}$ PEEK can maintain its mechanical properties at high temperatures, making it particularly useful for applications involving extreme highs of temperature.

Polycarbonates are already used in impact resistant safety applications such as protective eye wear and blast shields. ${ }^{19}$ It could be used to increase the impact resistance of a HAY and increase its demand for use in blast and impact resistant applications.

Like PTFE, acetal has a low coefficient of friction and can handle large loads due to its high stiffness value. $^{20}$ Used as a core it could provide a blanket structure to cover pipes to protect them from impacts. It could also provide a longer lifespan due to the limited abrasion between the wrap and core.

Two sets of analytical calculations were presented, as listed in Table 1. Set 1 presents analytical configuration with associated seven core materials and a nitinol as wrap material (with smaller core and wrap dimensions), whereas, Set 2 presents analytical configuration with an associated core material (rubber) and a nitinol as wrap material (with larger core and wrap dimensions). In both sets, the Poisson's ratio of the core, $\nu_{c}$, was varied between 0.49 and 0.35 , while all other variables were kept constant. Potential core materials have been added to the table to represent each increment of Poisson's ratio. 
Table I. Set I and 2: Analytical configuration with associated core materials and nitinol as wrap material (Poisson's ratio reference: rubber, ${ }^{21}$ polyurethane, ${ }^{22}$ polytetrafluoroethylene, ${ }^{23}$ polypropylene, ${ }^{24}$ polyetheretherketone, ${ }^{25}$ polycarbonate, ${ }^{26}$ acetal, ${ }^{27}$ nitinol ${ }^{28}$ ).

\begin{tabular}{|c|c|c|c|c|c|c|c|}
\hline Sets & Model no. & Core materials & $\begin{array}{l}\text { Core radius, } \\
R_{\circ}(\mathrm{mm})\end{array}$ & $\begin{array}{l}\text { Wrap dia., } \\
d_{\circ}(\mathrm{mm})\end{array}$ & $\begin{array}{l}\text { Wrap angle } \\
\left(\theta_{\circ}\right),{ }^{\circ}\end{array}$ & $\begin{array}{l}\text { Core Poisson's } \\
\text { ratio }\left(\nu_{c}\right)\end{array}$ & $\begin{array}{l}\text { Wrap Poisson's } \\
\text { ratio }\left(\nu_{w}, \text { nitinol }\right)\end{array}$ \\
\hline Set I & $\begin{array}{l}1 \\
2 \\
3\end{array}$ & $\begin{array}{l}\text { Rubber } \\
\text { Polyurethane (PU) } \\
\text { Polytetrafluoroethylene } \\
\text { (PTFE) (Teflon) }\end{array}$ & $\begin{array}{l}0.26 \\
0.26 \\
0.26\end{array}$ & $\begin{array}{l}0.13 \\
0.13 \\
0.13\end{array}$ & $\begin{array}{l}30 \\
30 \\
30\end{array}$ & $\begin{array}{l}0.49 \\
0.475 \\
0.45\end{array}$ & $\begin{array}{l}0.33 \\
0.33 \\
0.33\end{array}$ \\
\hline & $\begin{array}{l}4 \\
5\end{array}$ & $\begin{array}{l}\text { Polypropylene (PP) } \\
\text { Polyetheretherketone } \\
\text { (PEEK) }\end{array}$ & $\begin{array}{l}0.26 \\
0.26\end{array}$ & $\begin{array}{l}0.13 \\
0.13\end{array}$ & $\begin{array}{l}30 \\
30\end{array}$ & $\begin{array}{l}0.425 \\
0.4\end{array}$ & $\begin{array}{l}0.33 \\
0.33\end{array}$ \\
\hline & $\begin{array}{l}6 \\
7\end{array}$ & $\begin{array}{l}\text { Polycarbonate (PC) } \\
\text { Acetal }\end{array}$ & $\begin{array}{l}0.26 \\
0.26\end{array}$ & $\begin{array}{l}0.13 \\
0.13\end{array}$ & $\begin{array}{l}30 \\
30\end{array}$ & $\begin{array}{l}0.375 \\
0.35\end{array}$ & $\begin{array}{l}0.33 \\
0.33\end{array}$ \\
\hline Set 2 & 1 & Rubber & 4.9 & 0.375 & 30 & 0.49 & 0.33 \\
\hline
\end{tabular}

\section{Experimental work}

As per Set 2 analytical configuration with rubber as core and nitinol as wrap materials (Table 1), three HAY specimens were constructed with the same geometry using rubber and nitinol for the core and wrap, respectively. HAY specimen's fabrication for experimental testing was achieved through hand lay-up method. This included holding and tightening the end of the core at both ends using ties. Following which the wrapping wire was positioned at one end of core and wrapped along the core using a repeating helical pattern ensuring the wrap angle in every helix. This repeating helix formation until wrapping wire reached the other end of the core ultimately formed the HAY structure. As can be seen, the rubber used was of $9.8 \mathrm{~mm}$ diameter, whereas the commercial nitinol wire used was of $0.375 \mathrm{~mm}$ diameter $\left(\right.$ Flexinol $^{\circledR}$ Muscle Wire ${ }^{\circledR}$ ). The gauge length of each specimen was $210 \mathrm{~mm}$, but the total length was $320 \mathrm{~mm}$ to allow for the $60 \mathrm{~mm}$ depth of the chucks at either end. The nitinol was cut to a length of $375 \mathrm{~mm}$ which yielded six pitches at a wrap angle of $30^{\circ}$. Only the very ends of the wrap and core were bonded together (Figure 3(a)), using 3 $\mathrm{M}^{\mathrm{TM}}$ ScotchWeld $^{\text {TM }}$ Epoxy Adhesive DP190 Gray which still allowed movement between the wrap and core under tension (as the epoxy had high performance with high shear and peel strength, with plastic to metal bonding suggested applications and high mechanical properties (up to $30 \%$ elongation and $3500 \mathrm{psi}$ or $24 \mathrm{MPa}$ tensile strength)). ${ }^{29}$ As an additional measure care was taken when securing the HAY within the chucks to ensure that at least one of the bolts was tightened directly onto the wire (Figure 3(b)), to ensure it was secure.

An Instron ${ }^{\circledR} 3382$ (loading capacity: $100 \mathrm{kN}$ ) was used in conjunction with Bluehill ${ }^{\circledR}$ software to perform the tensile test at a strain rate of $30 \mathrm{~mm} / \mathrm{min}$ with data sampling rate of $10 \mathrm{~Hz}$. The test was set to stop at a final strain of 0.4 giving a final extension of $80 \mathrm{~mm}$. Bluehill $^{\circledR}$ was set-up to automatically record values for load and extension, (from which axial strain can be calculated), and an iPhone ${ }^{\circledR} 6 \mathrm{~s}$ was used to record the deformation in Ultra HD 4k. The experimental set-up is shown in Figure 3(c).

The test was repeated for each specimen so that mean values for strain and subsequently Poisson's ratio could be calculated and compared. Image analysis software ImageJ (open source image processing programme) was used to measure the transverse strain of the HAY at different axial strains throughout the duration of the video, thus enabling the Poisson's ratio to be calculated. To carry out this analysis a screenshot of the HAY under tensile loading was taken from a point in the video and then imported into Image $J$ where a pixel $/ \mathrm{mm}$ scale was established. The wide diameter of the chuck used to hold the HAY in the Instron ${ }^{\circledR} 3382$ was measured to be $45 \mathrm{~mm}$ using Sealey ${ }^{\circledR}$ digital callipers which gave an equivalent size of 97.7 pixels in ImageJ, hence a scale of 2.171 pixels $/ \mathrm{mm}$ was obtained. With the scale set, the gauge length of $210 \mathrm{~mm}$ was subtracted from the elongated HAY's length (using Instron $^{\circledR}$ 3382) to establish the extension. The transverse deformation of the HAY was measured in the same manner as seen in Figure 3(d) using ImageJ. These measurements were then used with following equations to determine the Poisson's ratio of the HAY. For example, at 0.151 axial strain a NPR of -1.471 was found:

$$
\begin{aligned}
& \varepsilon_{x}=\frac{\Delta L}{L_{0}}=\frac{241.81-210}{210}=0.151 \\
& \varepsilon_{y}=\frac{\Delta \emptyset}{\emptyset_{0}}=\frac{12.90-10.55}{10.55}=0.223 \\
& \nu_{x y}=-\frac{\varepsilon_{y}}{\varepsilon_{x}}=-\frac{0.223}{0.151}=-1.471
\end{aligned}
$$

where, $\Delta L$ is the tensile loading displacement applied in the boundary conditions and $L_{0}$ is the original length, and $\Delta \varphi$ is the change in lateral dimension and $\varphi_{0}$ is the original lateral dimension of the HAY, respectively. The process was repeated for different axial strains to 


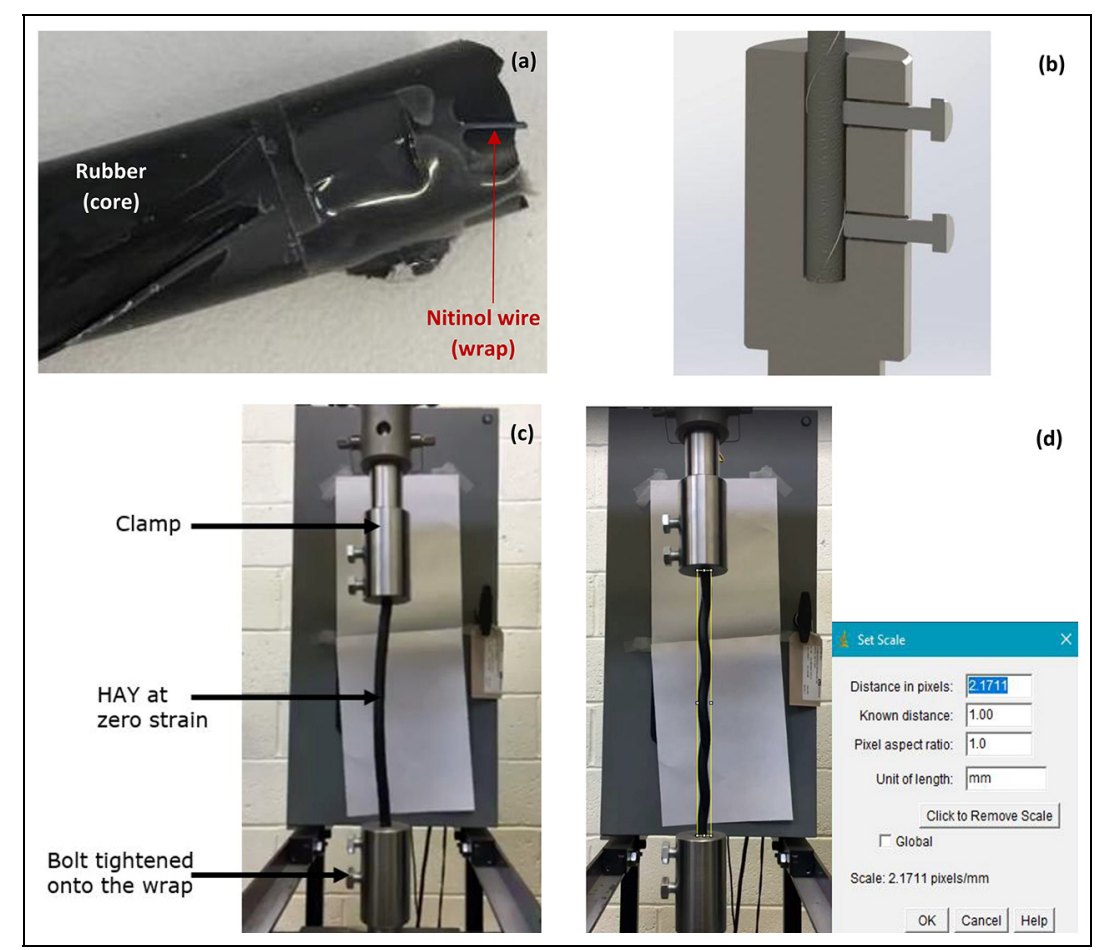

Figure 3. Experimental set-up: (a) Scotch-Weld ${ }^{\mathrm{TM}}$ epoxy structural DPI90 adhesive bonding between the rubber and the nitinol, (b) cross-section of chuck with the bolt tightened onto the wrap, (c) tensile loading experimental layout and (d) using ImageJ to measure extension and lateral expansion of HAY.

allow a graph of Poisson's ratio against axial strain to be generated for each specimen. The pictures with the corresponding axial strains can be seen in Figure 4 in sequential order (an example shown for experiment 1).

\section{Results}

\section{Analytical calculations with selected HAYs configurations in Set I}

With smaller core and wrap dimensions, as per Set 1 analytical configuration with associated core materials and nitinol as wrap material, Figure 5(a) (as an example) shows the relationship between the effective diameter and axial strain when $\nu_{c}=0.49$ (for rubber). As presented in Supplemental Appendix A (refer Supplemental Material), for each HAY material combination, the effective diameter $\left(D=\operatorname{Max}\left(D_{1}, D_{2}\right)\right)$ was plotted for the Stage 1 against the HAY axial strain between the limits of 0 and $\varepsilon_{c r i}$. Following this the effective diameter for the Stage $2\left(D=2\left(R_{2}+r_{2}\right)\right)$ was plotted against the HAY axial strain between the limits of $\varepsilon_{c r i}$ and the strain at which effective diameter fell back below the initial effective diameter. Figure 5(a) shows the effective diameter of model\#1 (i.e. rubber) throughout Stages 1 and 2 .

Figure 5(a) shows that at zero strain the effective diameter is equal to $0.78 \mathrm{~mm}$, then as strain is applied the HAY experiences initial contraction of the effective diameter as the helical path of the wrap fibre decreases, at 0.07 axial strain the wrap fibre has sufficiently displaced the core fibre so that expansion occurs at this point. This expansion occurs until the axial strain is equal to the critical strain where the wrap fibre lies straight in the centre of the HAY and the core cannot be displaced any further. With the wrap fibre now being elongated the effective diameter is seen to decrease linearly until the effective diameter reaches $0.76 \mathrm{~mm}$ at an axial strain of 0.65 . With the effective diameter, $D$, throughout the second stage tensile process calculated using equation (A.21) (Supplemental Appendix A (refer Supplemental Material)), the transverse strain, $\varepsilon_{y}$, was calculated using the equation:

$$
\begin{aligned}
\varepsilon_{y} & =\frac{\text { EffectiveDiameter }- \text { InitialEffective Diameter }}{\text { InitialEffectiveDiameter }} \\
& =\frac{D-2\left(R_{0}+d_{0}\right)}{2\left(R_{0}+d_{0}\right)}
\end{aligned}
$$

Using the ratio of transverse to axial strain, the Poisson's could be plotted throughout the whole tensile process for each HAY model (as per Set 1 analytical configurations, Table 1) and is shown overleaf in Figure 5(b).

From Figure 5(b) it is seen that each HAY model reacts the same way initially when under strain, up to an axial strain of 0.07 . At this point, there is seen to be slight differences in the amount the effective diameter changes as the axial strain approaches the critical strain. All models except for rubber and polycarbonate are 


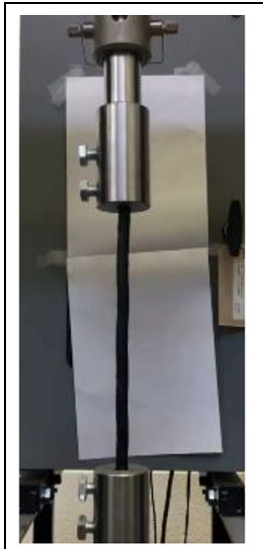

(a)

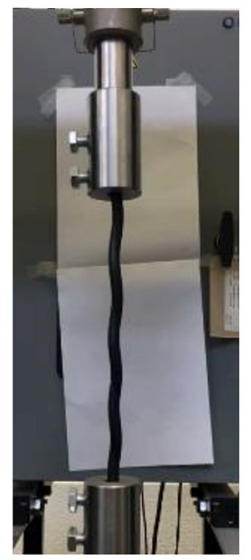

(e)

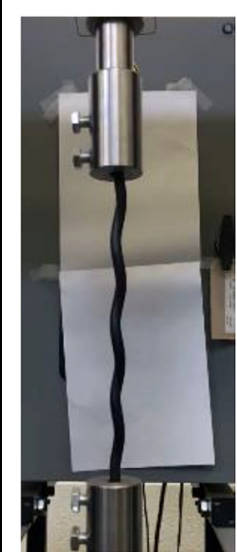

(i)

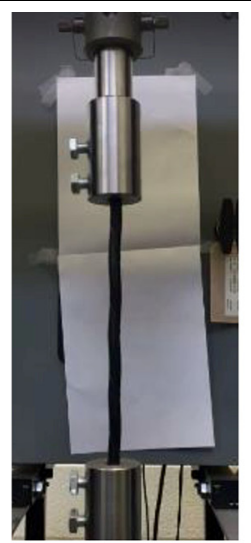

(b)

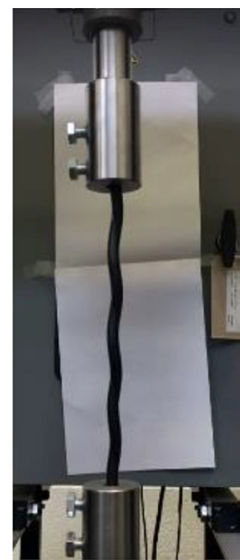

(f)

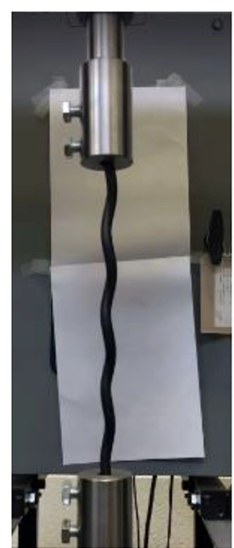

(j)

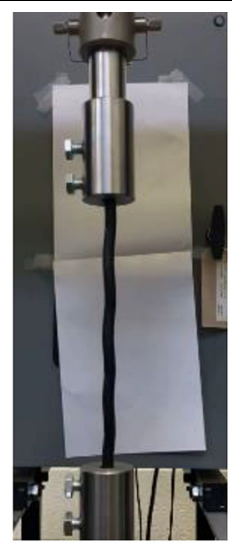

(c)

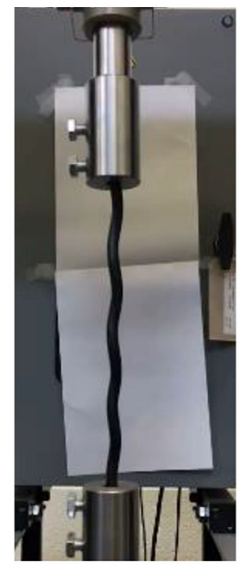

(g)

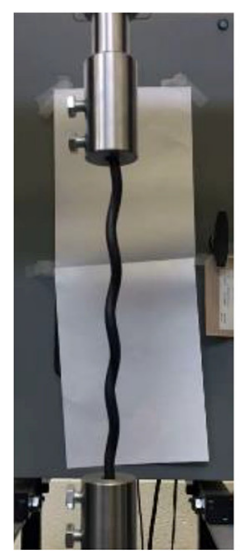

(k)

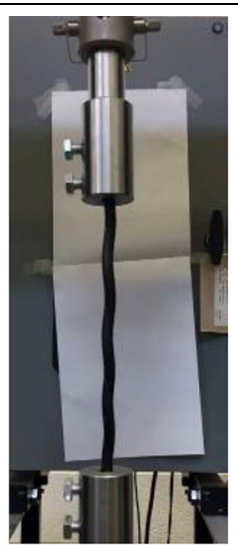

(d)

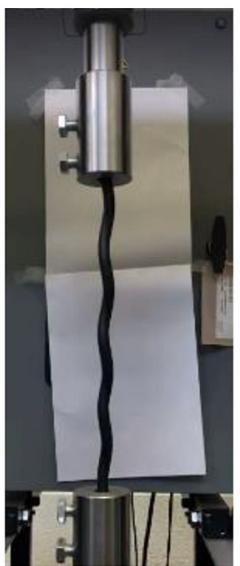

(h)

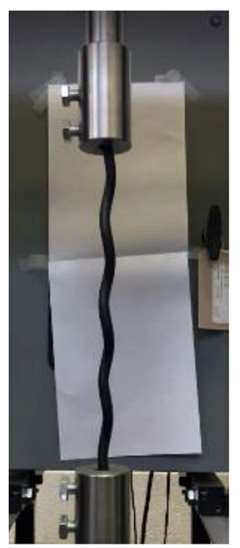

(1)

Figure 4. Pictures with the corresponding axial strains (experiment I) shown here in sequential order (a) to (l): (a) 0.015 , (b) 0.035 , (c) 0.062, (d) 0.09, (e) 0.12, (f) 0.13, (g) 0.15, (h) 0.18, (i) 0.2, (j) 0.22, (k) 0.24 and (l) 0.27.

seen to decrease in Poisson's ratio at 0.07 axial strain, whereas rubber and polycarbonate are seen to increase slightly before decreasing with a similar trend to the rest. All models reach their maximum NPR at the same axial strain, as expected due to the initial assumption used by this model. The Poisson's ratio of each model then approaches 0 although they achieve this at different axial strains with rubber achieving this at the lowest axial strain 0.65 and acetal achieving this at the highest strain of 0.9. The maximum NPR achieved by each model was then plotted against the Poisson's ratio of each core material and is shown in Figure 5(c).

Figure 5(c) also shows a clear trend between the core Poisson's ratio and the maximum NPR. With the exclusion of polycarbonate, the maximum NPR is seen to increase as the core's Poisson's ratio decreases. The HAY with rubber as the core, with the largest Poisson's ratio of 0.49 , exhibits a maximum NPR of -2.002 , whereas, acetal, with the lowest Poisson's ratio of 0.35 shows a maximum NPR increase of $16.9 \%$ at -2.341 . 


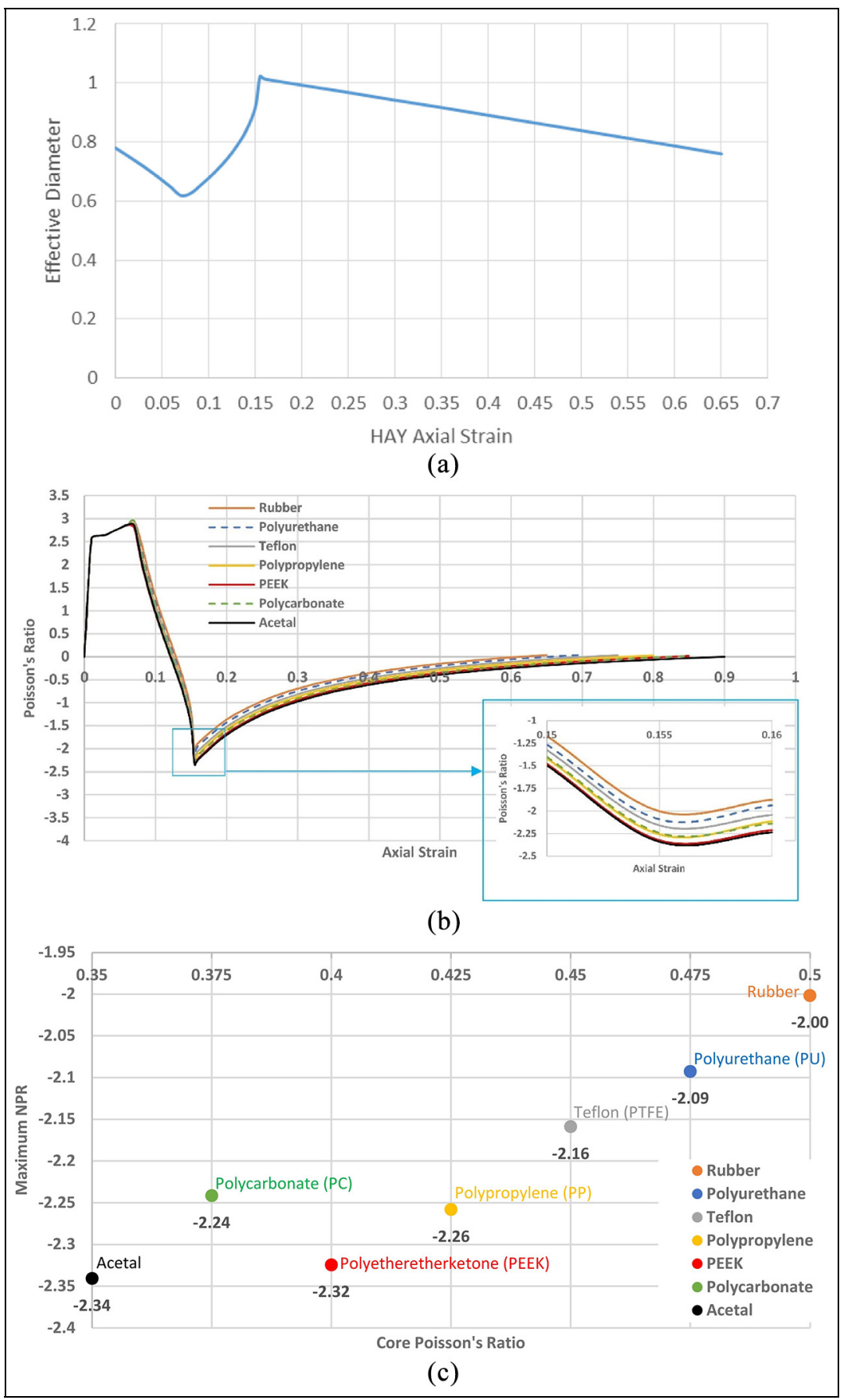

Figure 5. Set I analytical configurations: (a) predicted effective diameter of model I (core: rubber, wrap: nitinol) against HAY axial strain, (b) predicted Poisson's ratio for each HAY model throughout the tensile process, and (c) maximum NPR as the Poisson's ratio of the core is varied.

\section{Analytical calculations with selected HAYs configurations in Set 2}

With larger core and wrap dimensions, as per Set 2 analytical configuration with associated core materials and nitinol as wrap material, Figure 6(a) shows the relationship between the effective diameter and axial strain when $\nu_{c}=0.49$ (rubber of $9.8 \mathrm{~mm}$ diameter). The axial strain was plotted up to the point the effective diameter fell back below the initial effective diameter, which was seen to occur at an axial strain of 0.9 . Using transverse strain $\left(\varepsilon_{y}\right)$ equation shown above, the transverse strain was found, and hence the Poisson's ratio was calculated throughout the tensile process.

Figure 6(b) shows the predicted Poisson's ratio of the experimental HAY against the axial strain. As shown in Figure 6(b), the initial contraction of the HAY is seen to produce a Poisson's ratio of 3 , before beginning to expand. As the wrap angle was kept the 


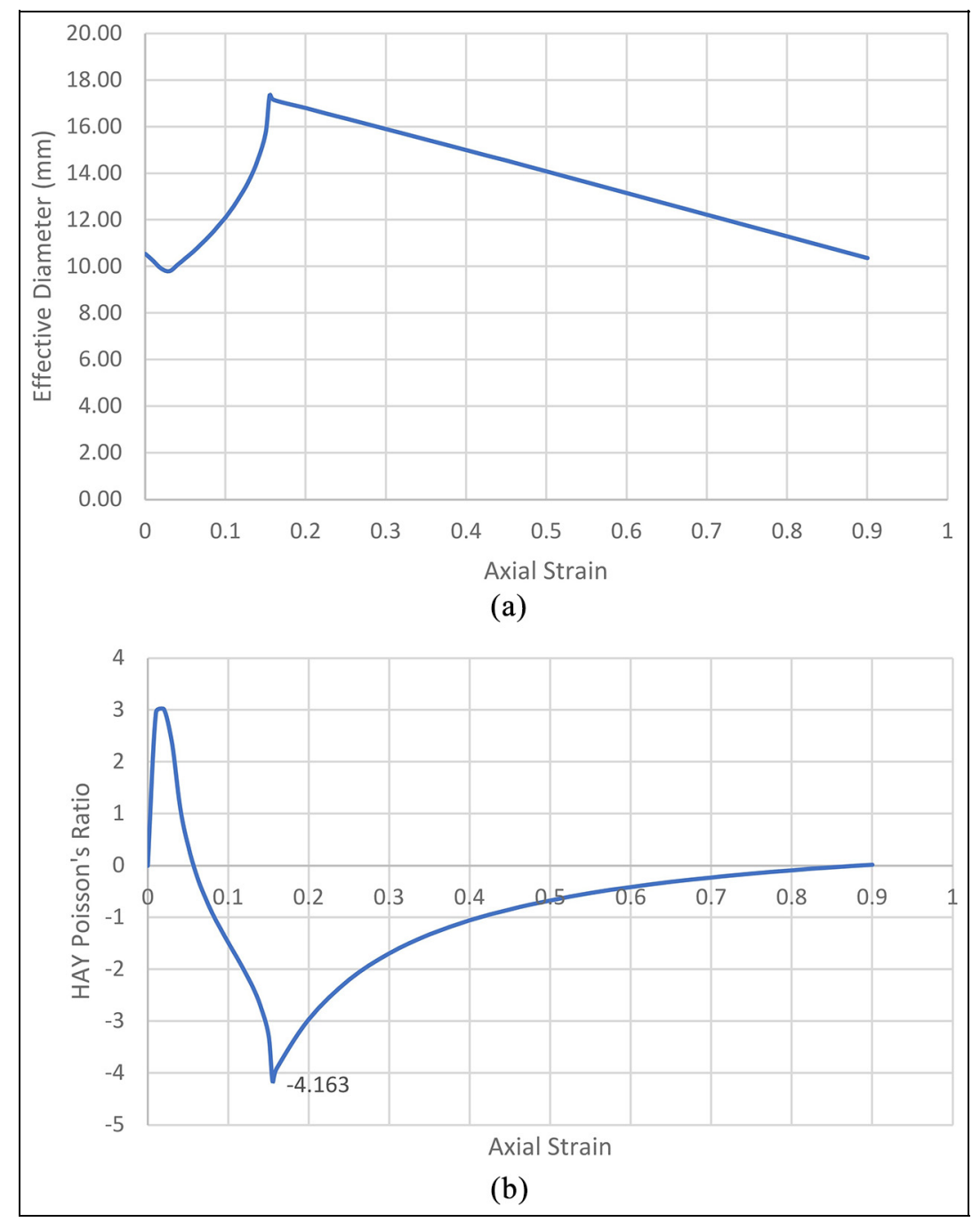

Figure 6. Set 2 analytical configuration: (a) predicted effective diameter of model I (core: rubber, wrap: nitinol) against HAY axial tensile strain and (b) predicted Poisson's ratio for of model I (core: rubber, wrap: nitinol) throughout the tensile process.

same (i.e. $30^{\circ}$ ) the maximum NPR occurs at the same point, where theory predicts this maximum to be -4.163 . From here the Poisson's ratio follows the gentle slope back towards 0 at an axial strain of 0.9 .

\section{Experimental tensile testing on HAYs}

The tensile tests on the three HAYs produced mixed results, as per Set 2 analytical configuration with rubber as core and nitinol as wrap materials (Table 1). Plots of load against extension for experiment 1,2 and 3 are presented in Figure 7(a). Experiment 1, yielded the best results as seen by a smooth increase in load and the immediate reduction in load 256-96 $\mathrm{N}$ that occurred when the wrap failed at an extension of $63 \mathrm{~mm}$. The second HAY (Experiment 2) to be tested produced a similar trend, to the previous experiment except for a dropin load at an extension of $59 \mathrm{~mm}$. The load increased further to $254 \mathrm{~N}$ after this point before the wrap failed at a final extension of $66 \mathrm{~mm}$. Results for the experiment 3, correlate well with the previous two for the first $30 \mathrm{~mm}$ of extension. Here at a load of $109 \mathrm{~N}$ the trend loses its linearity as the wrap began to slip until a final extension of $77 \mathrm{~mm}$ where the wrap is no longer held by the chuck.

As compared between the three tests (Figure 7(a)), in addition to highlighting the similar trend observed up to $30 \mathrm{~mm}$ extension for all tests, it also shows that for experiments 1 and 2 the wrap failed at almost the same load but different extensions. From Figure 7(a) it was also possible to calculate the strain energy stored radially in the lateral expansion of the HAY compared with the wrap and core components individually. The energy stored for each HAY is the difference between the energy stored before and after failure and the results are presented in Table 2 (Note: Energy stored before failure $\left(W_{B}\right)$ means work at breaking point, assuming a linear profile between origin and yield point; whereas energy stored after failure $\left(W_{A f t}\right)$ means work at breaking point, assuming a linear profile between origin and second phase loading). It was observed that the energy stored by specimen three was significantly lower than specimens 1 and 2 because the HAY lost structural integrity early in the test. The calculations used for 


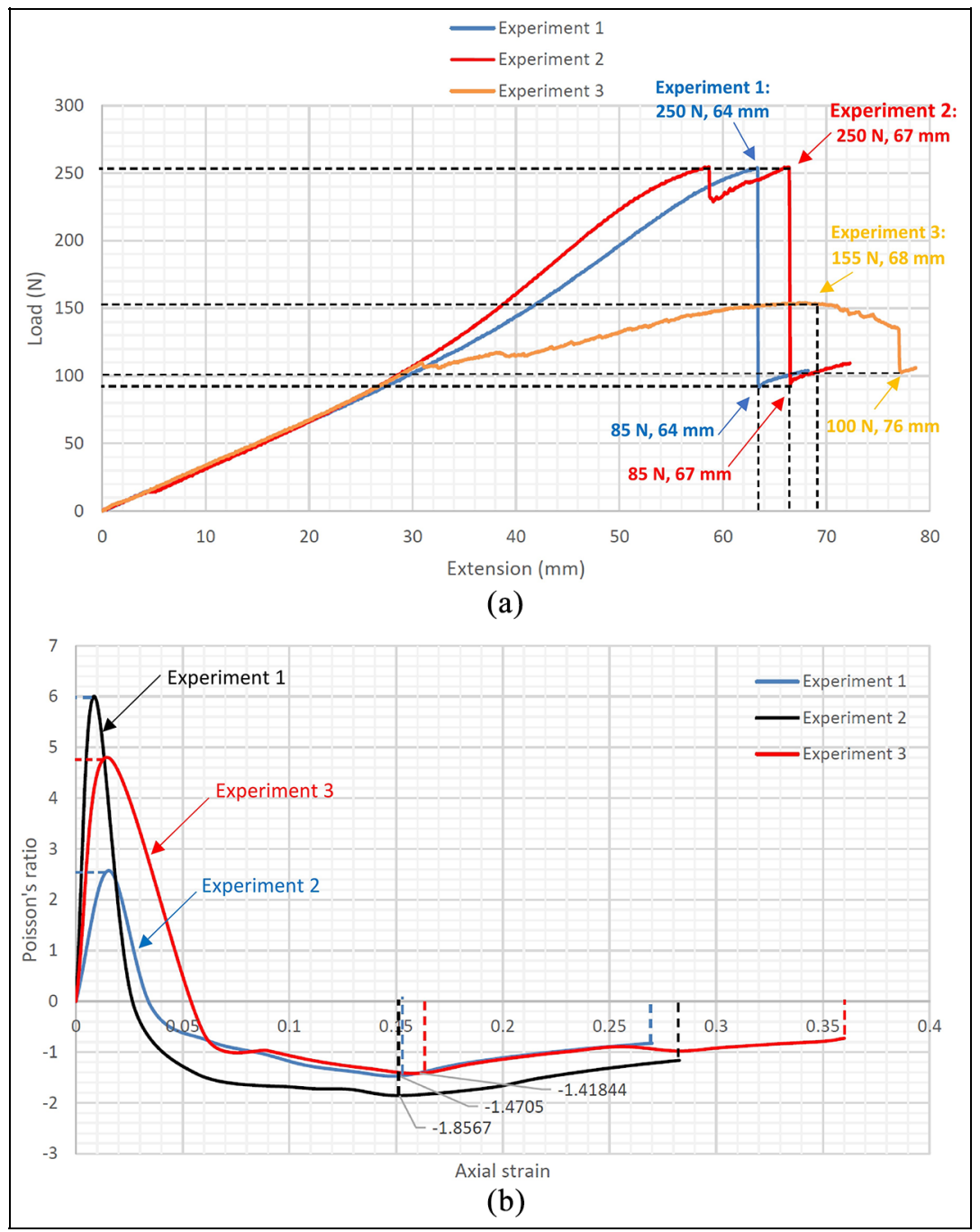

Figure 7. Comparison of the experiments I, 2 and 3 (three tensile testing on HAYs), as per Set 2 analytical configuration with rubber as core and nitinol as wrap materials: (a) load-extension profile and (b) calculated Poisson's ratio plotted against axial strain throughout the tensile process.

Table 2. Strain energy of a HAY (refer Supplemental Material, Appendix B).

\begin{tabular}{llll}
\hline Specimen & Before failure $(\mathrm{Nm})$ & After failure $(\mathrm{Nm})$ & Stored radially in the $\mathrm{HAY}(\mathrm{Nm})$ \\
\hline Experiment I & 8.00 & 2.72 & 5.23 \\
Experiment 2 & 8.37 & 2.84 & 5.52 \\
Experiment 3 & 5.27 & 3.80 & 1.47 \\
\hline
\end{tabular}

Table 2 can be found in Supplemental Appendix B (refer Supplemental Material).

Using the ImageJ technique to measure the transverse strain, the Poisson's ratio was calculated and plotted against axial strain for experiments 1,2 and 3 and can be seen in Figure 7(b). The general behaviour of Poisson's ratio as the axial strain increases correlates well with that found in literature. There is a clear maximum NPR of -1.471 at an axial strain of 0.151 . The trend from experiment 2 is like experiment 1 but there is a significantly higher rise in Poisson's ratio at low strain and a less obvious maximum NPR observed at an axial strain of 0.152 . Although the trend for experiment 3 is close to what was expected, there is more fluctuation in Poisson's ratio, particularly after the nitinol wrap begins to fail at an axial strain of 0.148 . The results yielded a clear representation of the discrepancies between results each of the three HAYs produced. The difference in auxetic behaviour exhibited by the three HAYs is clearly visible in Figure 7(b). Although the maxima and minima visible on the graph are significantly different, the trend however of Poisson's ratio 


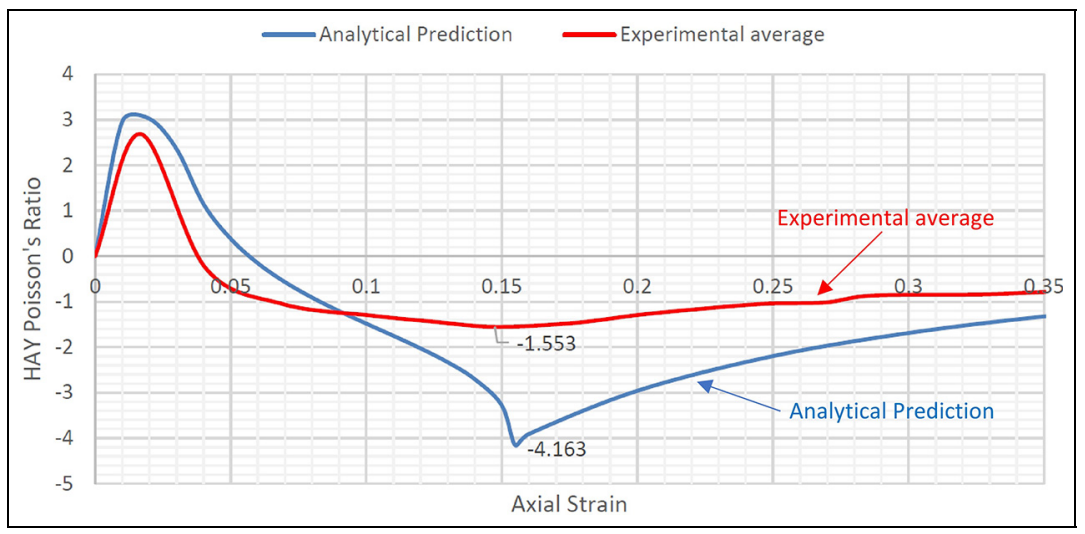

Figure 8. Comparison between the analytical prediction (as per Set 2 analytical configuration with rubber as core and nitinol as wrap materials) and the average from the three tensile experiments of HAYs.

as axial strain increases correlates well for the three experiments.

\section{Discussion}

\section{Analytical}

With smaller core and wrap dimensions, as per Set 1 analytical configuration with associated core materials and nitinol as wrap material, Figure 5(b), the Poisson's ratio predicted by the model increases during initial strain, reaches a maximum and then gradually decreases to the maximum NPR. For different core materials, the maximum NPR increases as the Poisson's ratio of the core decreases. For the core with a Poisson's ratio of 0.5 , as it experiences a tensile load, the core material will deform, and the diameter of the core material will become thinner (Figure 5(c)). As the Poisson's ratio of the core is decreased to 0.35 , the core has a higher resistance to transverse deformation as the tensile load is applied. Although the core will still deform and the diameter will become thinner, the magnitude of this deformation is less than that experienced by the core with a Poisson's ratio of 0.5 . Although in existing studies smaller wrap angles have been found to produce a greater maximum $\mathrm{NPR}^{9}$ the analytical model used here was found to be unreliable at smaller wrap angles. It is suggested that this is due to the wrap requiring less axial strain to be applied before it is fully extended.

\section{Experimental}

In general, the tensile tests carried out on the three HAYs (as per Set 2 analytical configuration with rubber as core and nitinol as wrap materials (Table 1)) were considered a success, especially for experiments 1 and 2. The analysis approach taken using ImageJ yielded results that correlated well with the trend predicted by the analytical model; however, the maximum NPR was significantly lower than expected. A comparison of results for the average from the three-tensile experimental and analytical studies (as per Set 2 analytical configuration with rubber as core and nitinol as wrap materials) is presented in Figure 8.

The mean Poisson's ratio for the three experiments (tensile tested samples shown in Figure 9(a)) as axial strain increased presented a similar trend to that predicted by the analytical model. At low axial strain, the Poisson's ratio increases due to an initial radial contraction of the HAY until the wrap become tight enough around the core to displace it laterally. At this point the Poisson's ratio decreases until the HAY becomes auxetic at axial strains of 0.041 and 0.057 from experimental and analytical methods, respectively. From the experiments, the maximum average NPR of -1.553 occurs at an axial strain of 0.146 which is slightly earlier than the critical strain of 0.155 predicted by theory. The differences occur because of the assumptions made as part of the analytical model ${ }^{5}$ and non-ideal experimental conditions. For example, friction is not accounted for analytically, but during the experiment it was obvious that there were significant frictional forces present between the nitinol wrap and rubber core. This was most evident during experiment 3 where the epoxy resin failed at the bottom half, but the HAY held its shape for some time after, shown in Figure 9(b). Figure 9(b) shows that even after the epoxy resin failed within the bottom chuck, the nitinol held its original helical path. The point at which the epoxy resin failed can be seen in Figure 9(c).

It was concluded that the structural integrity of the epoxy resin was impeded by the bolt being tightened directly onto it and creating a stress concentration. Similar observations were made for experiments 1 and 2 but the torque applied to the bolts was not as high. Ensuring the HAY was held under the same conditions for each experiment was one of the challenges that was faced. A pre-set torque tool should have been used to tighten the bolts to a known tension beforehand to increase consistency across the experiments. In addition, the length of the wrap held in the chuck $(60 \mathrm{~mm})$ could have been held with the epoxy resin to increase the adhesion surface area and reduce the risk of the wire losing contact with the core. 


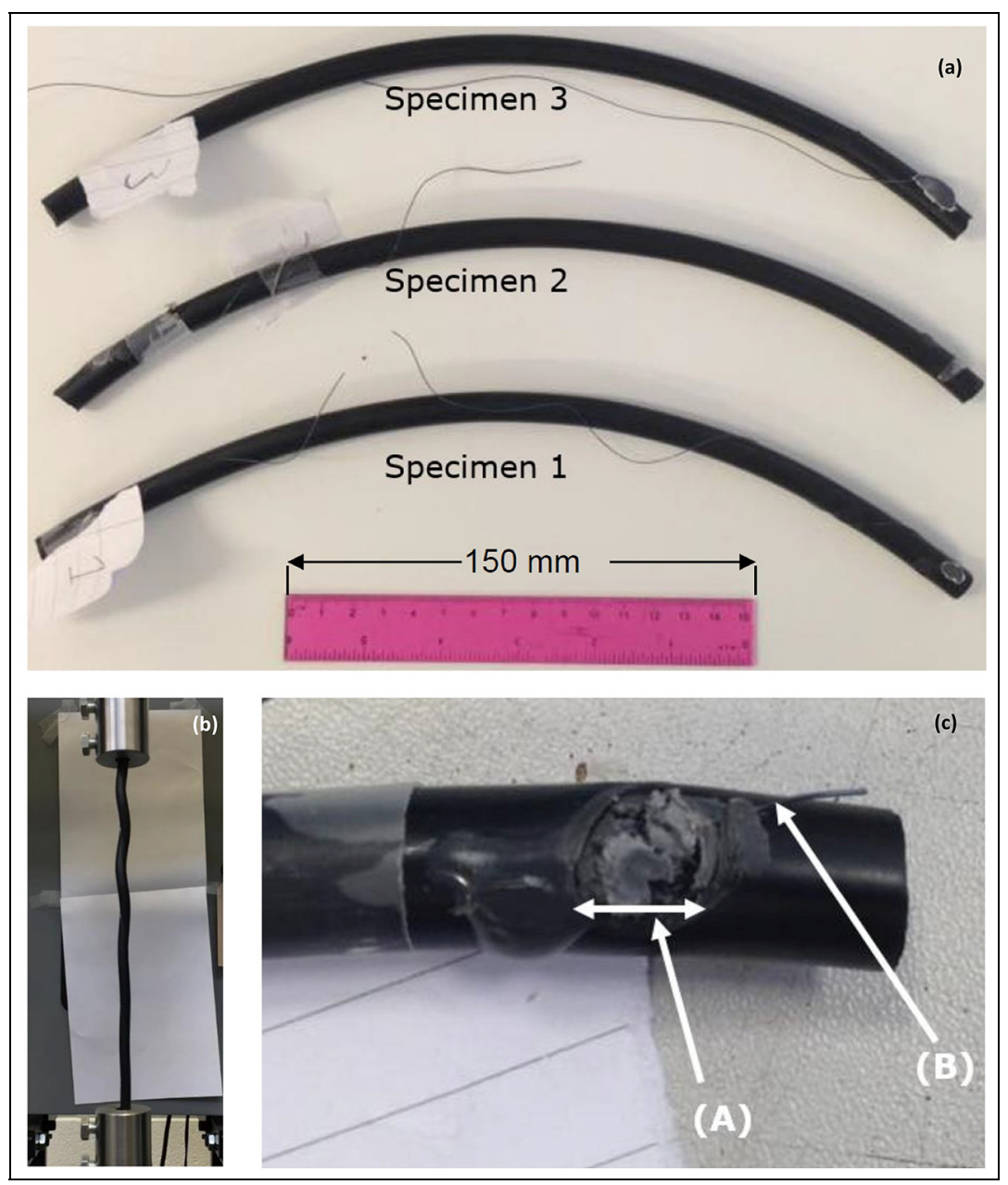

Figure 9. (a) All three tensile tested HAYs, (b) frictional forces after the epoxy resin failed and (c) failure of epoxy resin from experiment $3-(A)$ area subjected to stress from the bolt in the chuck (B) remaining part of the nitinol wire after failure.

It was observed during the experiments that the assumption made in the analytical model ${ }^{5}$ stating that no elongation occurred in the wrap until the critical strain (point at which the wrap is straight) does not hold true. The HAY far exceeded the critical strain and yet the wrap never became straight hence the core was not displaced to its full potential. Hence why the maximum NPR predicted by the analytical model is far larger than that obtained from the experiments.

Further differences between experimental and analytical results could be because the analytical model assumes the Young's modulus and Poisson's ratio remain constant for each material. Figure 7(a) shows the load against extension during the tensile test, from this figure experiment 1 exhibits stiffening at $30 \mathrm{~mm}$ extension before complete failure at $64 \mathrm{~mm}$, an equivalent axial strain of 0.142 and 0.31 , respectively. This limits the model in its capability to accurately predict the performance of the HAY as it does not account for the changing material properties of the wrap and core fibres and hence, will not predict the failure of either material.

The load against extension graphs for each HAY plotted together on Figure 7(a) highlights the differences between the three experiments (1,2 and 3). Experiment 1 produced the most definitive results as a drop in load yield point was not observed until the exact moment the nitinol wrap failed. The HAY of experiment 2 produced similar results to that of experiment 1 but a drop-in load was observed at an extension of $59 \mathrm{~mm}$. It was concluded that the drop was a result of the entire HAY slipping inside the chuck. After this point the load continued to increase before the nitinol wrap failed at a final extension of $66 \mathrm{~mm}$, equivalent to an axial strain of 0.314 . Results for experiment 3 were only reliable up to an extension of $30 \mathrm{~mm}$. It was at this point that the nitinol wrap began to slide out of the chuck. The result of this was that the load carrying capacity of the HAY was impeded because only the rubber core was carrying a load. It was concluded the HAY was slipping because the chucks used were not ideal. If too tightened excessively the small surface area of the bolt produced a large concentration of stress on the HAY resulting in failure as seen in experiment 3. Conversely if the bolt was not tightened enough, it would not grip the HAY adequately and it would inevitably slip as seen in experiment 2 . It is important to note that there will be effect of boundary conditions 
near chucks. Length near boundary region was not excluded in the current analysis (as it appears the effect of boundary conditions near clamps is minimal (as shown in Figure 4 (corresponding axial strains)). For future tests, different chucks that clamp around the HAY instead of being bolted on should be used to increase the contact surface area and reduce stress concentrations on the HAY.

Additional variations between the individual experiments can be put down to differences in construction of the HAY specimens. It was a challenge to recreate identical HAYs by hand and although the lengths were constant, it is unlikely that an exact wrap angle of $30^{\circ}$ was repeated for the three HAYs. However, this conforms to the theory that the initial wrap angle heavily influences the maximum NPR. Reading errors occurred when using the ImageJ approach for obtaining Poisson's ratio because it was difficult to interpret edge of the HAYs when zoomed in. A higher quality camera could have been used, or an automatic method of measuring the transverse deformation could have been developed.

The strain energy shown to be stored in the radial expansion of the HAY found in Table 2 shows that the auxetic behaviour of a HAY allows for more energy to be absorbed when subjected to a tensile load when compared to that stored by the individual components. After the nitinol wrap fails, a significant drop in load was observed in Figure 7(a), after which the load is being carried solely by the rubber core. It can be concluded that an auxetic structure provides increased energy absorption making it ideal for use in impact resistance applications. ${ }^{30}$

\section{Parametric analysis}

The parametric analysis carried out in this study focused primarily on changing the mechanical properties of the core component. Further analysis depth could be added by investigating the influence that the wrap material has on the NPR of the HAY while keeping the core material the same, ${ }^{31}$ thus leading to an investigation into different combinations of component materials. Combining this with existing work would enable the optimal geometric and mechanical properties of the HAY to be identified, creating new opportunities for using HAYs in different environments (e.g. marine structure safety applications where development of corrosion resistant composites may be necessary), or blast resistant curtains, impact resistance applications in automobiles or structures. ${ }^{32,33}$ The possible applications of HAYs greatly increase when considering their potential use within $2 \mathrm{D}$ and $3 \mathrm{D}$ structures. While the auxetic behaviour of an individual HAY is well understood, few studies (experimental, theoretical or FEA) have been conducted where HAYs have been woven together to create a carpet or sponge like structure. It would be beneficial to understand how said structures react to compressive/tensile loading and impact testing to further explore their potential.

\section{Conclusion}

The aim of this study was to investigate the influence of core materials on the mechanical performance of HAY when using the nitinol as a wrap. Possible applications for HAYs made of light-weight materials such as PEEK or PTFE could replace conventional structures have been identified. As advancements in understanding auxetic behaviour are made, the potential to control and optimise the NPR will increase leading to new possibilities for this technology to be applied. It is concluded that:

a. Through analytical studies it was observed that core materials with a higher Poisson's ratio yielded a greater maximum NPR. This is because at a higher Poisson's ratio the core will deform more readily when interacting with the wrap as axial strain is increased. It should be noted that a decrease of $30 \%$ in core Poisson's ratio resulted in an increase in NPR of only $16.9 \%$. The analytical model predicted that the maximum NPR would occur when the wrap became entirely straight, that is, the critical strain.

b. Although the maximum NPR for all three experiments occurred at an axial strain within $4.5 \%$ of the critical strain, it was observed that the deformed wrap angle $\left(\theta^{\prime}\right)$ had not reduced to zero as predicted analytically. The maximum NPR from the three experiments was significantly less than theory had predicted, leading to a suggestion that frictional forces between the HAY components significantly impedes the maximum potential NPR.

c. The use of a nitinol wrap is expected to increase the maximum NPR of a HAY compared with conventional materials such as stainless steel or carbon fibre. Materials with low coefficients of friction such as PTFE or acetal would enhance the auxetic behaviour if used as for the core material.

\section{Acknowledgements}

The authors acknowledge the support of Dr Jennifer McConnachie as project co-supervisor (now retired), $\mathrm{Mr}$ Allan MacPherson (Engineering Design Technician) and Dr Kristof Starost to carry the experimental work, and Mr Sean Smith, assisting in the development of the 3D Solidworks model of the HAY carpet, all at the School of Engineering, Robert Gordon University.

\section{CRediT authorship contribution statement}

Nadimul Faisal: Conceptualisation, Supervision, Data curation, Writing - Original and final draft preparation, reviewing 
and editing. Andrew Fowlie: Investigation, Testing, Validation, Reporting. Joe Connell: Investigation, Testing, Validation, Reporting. Sean Mackenzie: Investigation, Testing, Validation, Reporting. Ryan Noble: Investigation, Testing, Validation, Reporting. Anil Prathuru: Experimental support, reviewing and editing.

\section{Declaration of conflicting interests}

The author(s) declared no potential conflicts of interest with respect to the research, authorship, and/or publication of this article.

\section{Funding}

The author(s) received no financial support for the research, authorship, and/or publication of this article.

\section{ORCID iD}

Nadimul Haque Faisal (D) https://orcid.org/0000-0001-50336336

\section{Supplemental material}

Supplemental material for this article is available online.

\section{References}

1. Wright JR, Burns MK, James E, et al. On the design and characterisation of low-stiffness auxetic yarn and fabrics. Text Res $J$ 2012; 82: 645-654.

2. Bhattacharya S, Zhang GH, Ghita $\mathrm{O}$, et al. The variation in Poisson's ratio caused by the interactions between the core and wrap in helical auxetic yarns. Compos Sci Technol 2014; 102: 87-93.

3. Zhang GH, Ghita O and Evans KE. The fabrication and mechanical properties of a novel 3-component auxetic structure for composites. Compos Sci Technol 2015; 117: 257-267.

4. Du Z, Zhou M, He L, et al. Study on negative Poisson's ratio of auxetic yarn under tension: Part 2 - experimental verification. Text Res $J$ 2015; 85: 768-774.

5. Du Z, Zhou M, Liu H, et al. Study on negative Poisson's ratio of auxetic yarn under tension: Part 1 - theoretical analysis. Text Res $J$ 2015; 85: 487-498.

6. Zhang G, Ghita OR and Evans KE. Dynamic thermomechanical and impact properties of helical auxetic yarns. Compos Part B 2016; 99: 494-505.

7. Nazir MU, Shaker K, Hussain R, et al. Performance of novel auxetic woven fabrics produced using Helical Auxetic Yarn. Mater Res Express 2019; 6: 085703.

8. Ullah T, Ahmad S and Nawab Y. Development of helical auxetic yarn with negative Poisson's ratio by combinations of different materials and wrapping angle. $J$ Ind Text. Epub ahead of print 9 July 2020. DOI: 10.1177/ 1528083720941116.

9. Zhang GH, Ghita O, Lin C, et al. Varying the performance of helical auxetic yarns by altering component properties and geometry. Compos Struct 2016; 40: 369-377.

10. Urdanpilleta $M$ and Sevillano JG. A novel method of analysis of superplastic behaviour. Mater Lett 2004; 58: 3052-3057.

11. Mahtabi MJ, Shamsaei N and Mitchell MR. Fatigue of nitinol: the state-of-the-art and ongoing challenges. $J$ Mech Behav Biomed Mater 2015; 50: 228-254.
12. Scarpa F, Giacomin J, Zhang Y, et al. Mechanical performance of auxetic polyurethane foam for antivibration glove applications. Cell Polym 2005; 24: 253-268.

13. Biswas KS and Vijayan K. Friction and wear of PTFE - a review. Wear 1992; 158: 193-211.

14. Evans KE and Alderson A. Auxetic materials: functional materials and structures for lateral thinking. Adv Mater 2000; 12: 617-628.

15. Evans KE and Alderson KL. Auxetic materials: the positive side of being negative. Eng Sci Educ J 2000; 9: 148-154.

16. Alderson KL, Webber RS, Kettle AP, et al. A novel fabrication route for auxetic polyethylene. part 1: processing microstructure. Polym Eng Sci 2005; 45: 568-578.

17. Textile World. The Auxetic Effect, http://www.textileworld.com/textile-world/quality-fabric-of-the-month/ 2010/05/the-auxetic-effect/ (2010, accessed 4 March 2017).

18. Regis M, Bellare A, Pascolini T, et al. Characterization of thermally annealed PEEK and CFR-PEEK composites: structure-properties relationships. Polym Degrad Stab 2017; 136: 121-130.

19. Sarva S, Mulliken AD and Boyce MC. Mechanics of taylor impact testing of polycarbonate. Int J Solids Struct 2007; 44: 2381-2400.

20. Dziadur W. The effect of some elastomers on the structure and mechanical properties of polyoxymethylene. Mater Charact 2001; 46: 131-135.

21. Matweb. Arlon Thermabond(R) 99950 N008 silicone rubber/fiberglass, http://www.matweb.com/search/datasheet. aspx?matguid $=$ a3c68698c89742dfb27e7877e02943df (2016, accessed November 2016).

22. Qi HJ and Boyce MC. Stress-strain behavior of thermoplastic polyurethane. Mech Mater 2005; 37: 817-839.

23. DuPont. Teflon(TM) PTFE properties handbook, http:// www.rjchase.com/ptfe_handbook.pdf (2015, accessed November 2016).

24. INEOS. Typical engineering properties of polypropylene, https://www.ineos.com/globalassets/ineos-group/busines ses/ineos-olefins-and-polymers-usa/products/technicalinformation-patents/ineos-engineering-properties-of-pp. pdf (2014, accessed November 2016).

25. Matweb. Victrex(TM) PEEK $150 \mathrm{G}$ easy flow, http:// www.matweb.com/search/datasheet.aspx?matgui$\mathrm{d}=096 \mathrm{a} 4 \mathrm{ba} 9 \mathrm{~b} 3 \mathrm{c} 840 \mathrm{~b} 29 \mathrm{~d} 2 \mathrm{e} 80108 \mathrm{a} 771 \mathrm{fcf}$ (2016, accessed November 2016).

26. Plastics International. Marklon GP polycarbonate, https://www.plasticsintl.com/datasheets/Polycarbonate.pdf (2016, accessed November 2016).

27. Matweb. DuPont performance polymers Delrin ${ }^{\circledR} 150 \mathrm{E}$, high viscosity acetal homopolymer, http://www.matweb. $\mathrm{com} / \mathrm{search} /$ datasheet.aspx?matguid $=35 \mathrm{bd} 15 \mathrm{a} 0 \mathrm{bf} 2 \mathrm{f} 4 \mathrm{e} 9880$ 5b23abe5d46bf7\&ckck $=1 \quad(2006$, accessed November 2016).

28. Technical characteristics of Flexinol ${ }^{\circledR}$ Muscle Wire ${ }^{\circledR}$, http://www.dynalloy.com/pdfs/TCF1140.pdf (accessed November 2016).

29. Scotch-Weld ${ }^{T M}$ Epoxy structural DP190 adhesive, http:// pdf2.datasheet.su/3m/dp-190-gray.pdf (accessed May 2021).

30. Darja R, Tatjana R and Alenka P-C. Auxetic textiles. Acta Chim Slov 2013; 60: 715-723.

31. McAfee $\mathbf{J}$ and Faisal NH. Parametric sensitivity analysis to maximise auxetic effect of polymeric fibre based helical yarn. Compos Struct 2017; 162: 1-12. 
32. Khan MI, Akram J, Umair M, et al. Development of composites, reinforced by novel 3D woven orthogonal fabrics with enhanced auxeticity. J Ind Text 2019; 49(5): 676-690.

33. Ali M, Zeeshan M, Qadir MB, et al. Development and mechanical characterization of weave design based 2D woven auxetic fabrics for protective textiles. Fib Polym 2018; 19(11): 2431-2438.

\section{Appendix}

\section{Notations}

\section{HAY}

NPR

$\mathrm{PC}$

PEEK

PP

PTFE

PU

$d$

$d_{2}$

$d_{0}$

D
Helical Auxetic Yarn

Negative Poisson Ratio

Polycarbonate

Polyetheretherketone

Polypropylene

Polytetrafluoroethylene

Polyurethane

Deformed Wrap Diameter

Deformed Wrap Diameter during the

Second Stage

Initial Wrap Diameter

Effective Diameter during the Second

Stage
Outer Contour Diameter of Wrap Fibre Outer Contour Diameter of Core Fibre Initial Length of Wrap per Pitch Helical Radius of the Core Fibre Axis Helical Radius of the Core Fibre Axis During the Second Stage Deformed Core Radius During the First Stage

$R_{2} \quad$ Deformed Core Radius During the Second Stage

$R_{0} \quad$ Initial Core Radius

$\alpha \quad$ Initial Core Angle

$\varepsilon_{c} \quad$ Core's Axial Strain During the First Stage

$\varepsilon_{c 2} \quad$ Core's Axial Strain During the Second Stage

$\varepsilon_{\text {crit }} \quad$ Critical Axial Strain

$\varepsilon_{w} \quad$ Wrap's Axial Strain

$\varepsilon_{t} \quad$ Axial Strain of HAY

$\theta^{\prime} \quad$ Deformed Wrap Angle

$\theta_{0} \quad$ Initial Wrap Angle

$\lambda \quad$ Initial Pitch

$\lambda^{\prime} \quad$ Deformed Pitch

$\nu_{c} \quad$ Core's Poisson ratio

$\nu_{w} \quad$ Wrap's Poisson ratio

$\nu+\max \quad$ Maximum Positive Poisson's ratio 


\section{Supplementary Material}

Investigating the influence of the core material on the mechanical performance of a nitinol wire wrapped helical auxetic yarn

Nadimul Haque Faisal ${ }^{1}$, Andrew Fowlie, Joe Connell, Sean Mackenzie, Ryan Noble, Anil Kumar Prathuru

School of Engineering, Robert Gordon University, Garthdee Road, Aberdeen, AB10 7GJ, UK

Appendix A: Analytical calculations (as per Du et al. [5])

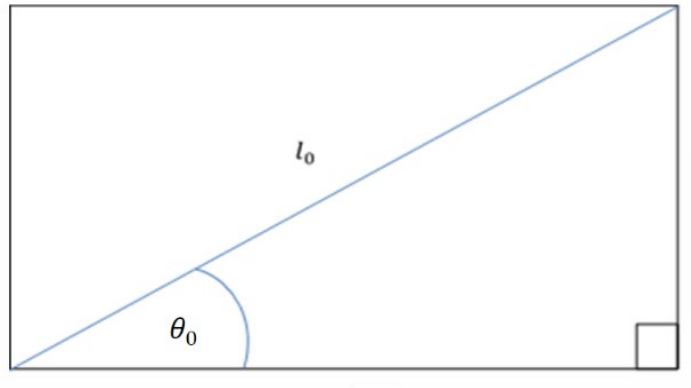

$\lambda$

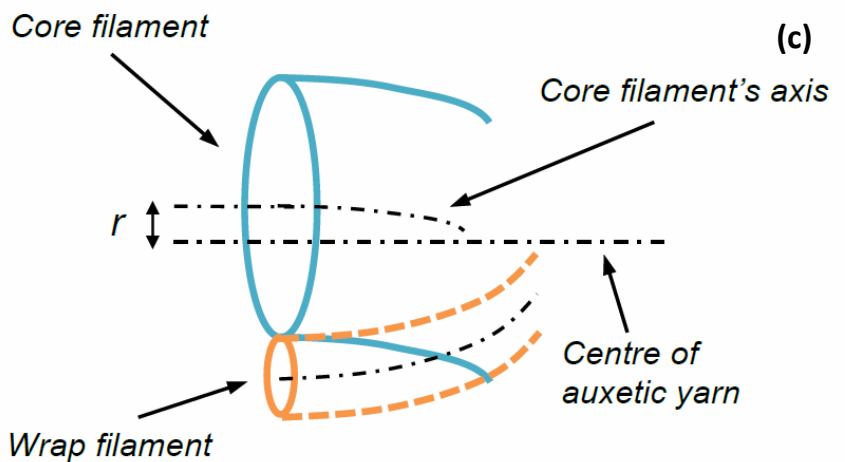

(a)

(c)
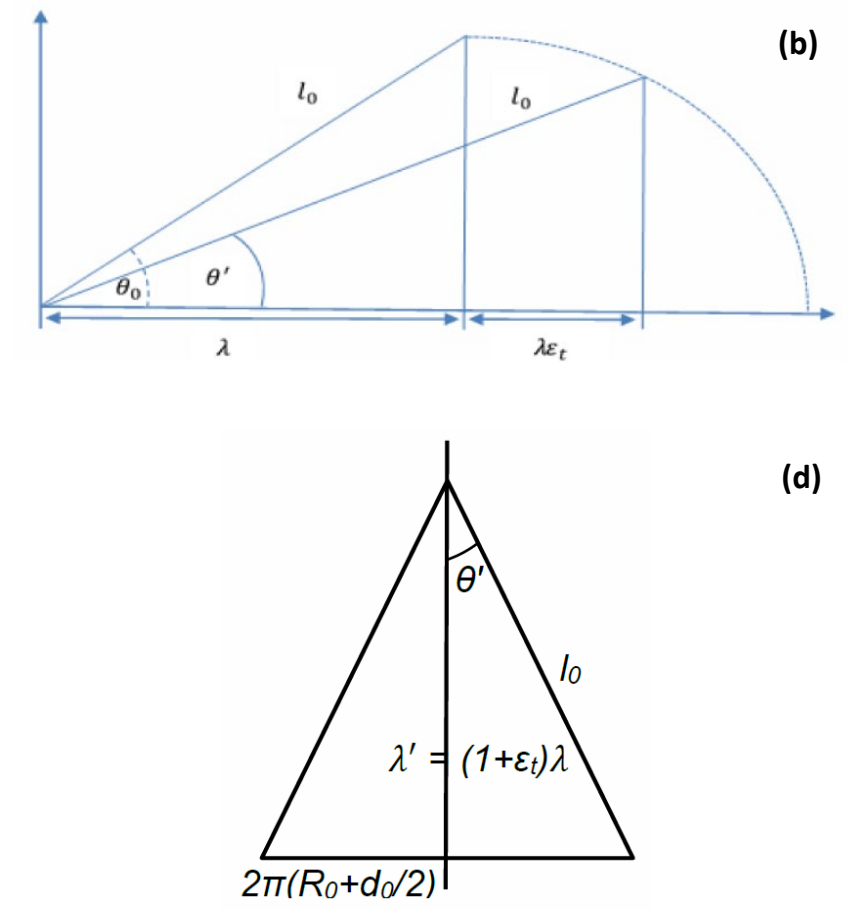

(d)

(e)

$$
l_{0}\left(1+\varepsilon_{w}\right)
$$

Fig. A.1. Stage 1: (a) helical path of wrap fibre, (b) deformed helical path of wrap fibre, (c) cross-sectional schematic diagram, (d) extended helical path of wrap fibre, and Stage 2: (e) helical path of the core filament.

\footnotetext{
${ }^{1}$ Corresponding authors. E-mail addresses: N.H.Faisal@rgu.ac.uk; Tel: +44 (0) 1224-26 2438.
} 
Stage 1: The critical strain $\left(\varepsilon_{c r i}\right)$ is the strain at which the wrap filament becomes straight in the centre of the HAY. This was derived by equation (A.1) by considering a single pitch of the wrap fibre as seen in Figure A.1(a) showing the relationship between the initial pitch length $\lambda$ and the length of wrap per pitch $l_{0}$, where $\theta_{0}$ is the initial wrap angle.

$\varepsilon_{c r i}=\frac{\frac{\lambda}{\cos \theta_{0}}-\lambda}{\lambda}=\frac{1}{\cos \theta_{0}-1}$

Upon elongation the wrap angle decreases to the deformed wrap angle $\theta^{\prime}$ until it lies in the centre of the HAY with an angle of $0^{\circ}$, and the pitch will change from $\lambda$ to $\lambda+\lambda \varepsilon_{t}$, where $\varepsilon_{t}$ is the axial strain of the HAY. Figure A.1(b) shows how the wrap angle changes when the HAY is under strain. Due the assumption 2 (i.e. the wrap fibre experiences no elongation until $\theta^{\prime}$ equals zero. From Figure A.1(b), equation (A.2) can be derived:

$\cos \theta^{\prime}=\frac{\lambda\left(1+\varepsilon_{t}\right)}{l_{0}}=\frac{\lambda\left(1+\varepsilon_{t}\right)}{\frac{\lambda}{\cos \theta_{0}}}=\left(1+\varepsilon_{t}\right) \cos \theta_{0}$

This can then be rearranged in equation (A.3) for deformed wrap angle:

$\theta^{\prime}=\cos ^{-1}\left[\left(1+\varepsilon_{t}\right) \cos \theta_{0}\right]$

As the wrap angle decreases, the core fibre deforms to the helical path of the wrap fibre as shown in Figure A.1(c). The distance from the central point of the core fibre's cross section to the centreline of the yarn is denoted as $r$ and is described as the helical radius of the core fibre axis. When the deformed wrap angle is $\theta^{\prime}$, the helical radius of the core fibre axis can be seen in equation (A.4), where $R$ is equal to the radius of the deformed core fibre and $d_{0}$ is the diameter of the core fibre.

$r=\left(R+\frac{d_{0}}{2}\right)-\frac{l_{0} \sin \theta^{\prime}}{2 \pi}$

Per the definition of Poisson's ratio, the radius of the core fibre deforms as a function of axial strain, equation (A.5), where $R_{0}$ is the initial radius of the core fibre, $v_{c}$ is the Poisson's ratio of the core fibre and $\varepsilon_{c}$ is the axial strain of the core fibre. 
$R=R_{0}-v_{c} \varepsilon_{c} R_{0}$

The axial strain of the core fibre is not equal to the equal to axial strain of the HAY. The axial strain of the core fibre can be derived from Figure A.1(d) by using Pythagoras's theorem to solve for $l_{0}$ and by then using the strain equation, the axial strain of the core fibre can be derived and expressed in equation (A.6):

$\varepsilon_{C}=\frac{\sqrt{\left(2 \pi\left(R_{0}+\frac{d_{0}}{2}\right)\right)^{2}+\lambda^{2}\left(1+\varepsilon_{t}\right)^{2}-\lambda}}{\lambda}$

By substituting equations (A.5) and (A.6) into equation (A.4) the helical radius of the core fibre axis can be expressed by equation (A.7):

$r=\left[R_{0}\left(1-v_{C} \frac{\sqrt{\left(2 \pi\left(R_{0}+\frac{d_{0}}{2}\right)\right)^{2}+\lambda^{2}\left(1+\varepsilon_{t}\right)^{2}-\lambda}}{\lambda}\right)+\frac{d_{0}}{2}\right]-\frac{l_{0} \sin \theta^{\prime}}{2 \pi}$

Using the $\operatorname{cosine}$ rule, $\sin \theta^{\prime}$ was found by solving for $\lambda$ in Figure A.1(a) and substituted into Figure A.1(d). Pythagoras's theorem was then used to solve for the lower edge of the triangle before using the sine rule to obtain equation (A.8):

$\sin \theta^{\prime}=\sqrt{1-\left(1+\varepsilon_{t}\right)^{2} \cos ^{2} \theta_{0}}$

Equations (A.9) and (A.10) were derived from Figure A.1(a) by using the sine rule and tangent rule respectively and then rearranged to obtain $l_{0}$ in equation (A.9) and $\lambda$ in equation (A.10):

$l_{0}=\frac{2 \pi\left(R_{0}+\frac{d_{0}}{2}\right)}{\sin \theta_{0}}$

$\lambda=\frac{2 \pi\left(R_{0}+\frac{d_{0}}{2}\right)}{\tan \theta_{0}}$

By substituting equations (A.8), (A.9) and (A.10) into equation (A.7) the final expression describing the helical radius of the core fibre axis is given in equation (A.11): 
$r=R_{0}\left(1-v_{c} \frac{\sqrt{4 \pi^{2}\left(R_{0}+\frac{d_{0}}{2}\right)^{2} \tan ^{2} \theta_{0}+\left[2 \pi\left(R_{0}+\frac{d_{0}}{2}\right)\right]^{2}\left(1+\varepsilon_{t}\right)^{2}}}{2 \pi\left(R_{0}+\frac{d_{0}}{2}\right)}-v_{c}\right)+\frac{d_{0}}{2}-\frac{\left(R_{0}+\frac{d_{0}}{2}\right) \sqrt{1-\left(1+\varepsilon_{t}\right)^{2} \cos ^{2} \theta_{0}}}{\sin \theta_{0}}$ (A.11)

The effective diameter is considered as the diameter that a cylinder would have to fully enclose the HAY. $D_{1}$ is the outer contour diameter of the wrap fibre, initially the effective diameter will be measured as the diameter of the imaginary enclosing cylinder. As strain is applied the helical radius of the wrap fibre will decrease to 0 as it approaches the critical strain. Hence, as strain is applied $D_{1}$ will decrease to the diameter of the wrap fibre $d_{0}$. The equation (A.12) describes the outer contour diameter:

$D_{1}=2\left(\frac{d_{0}}{2}+\frac{l_{0} \sin \theta^{\prime}}{2 \pi}\right)$

Similarly, to $D_{1}, D_{2}$ is equal to the outer contour diameter of the core fibre. The core fibre initially starts in the centre of the HAY, as strain is then applied the core is displaced by the wrap and the outer contour diameter will then increase until it reaches its maximum at the critical strain. The equation for the outer contour diameter of the core fibre $D_{2}$ is shown in equation (A.13):

$D_{2}=2(R+r)$

The effective diameter will experience initial contraction before changing the point of measurement to the outer surfaces of the core fibre where it will then expand. Therefore, the effective diameter is described as the maximum value of $D_{1}$ and $D_{2}$ throughout the tensile process and can be seen in equation (A.14):

$D=\operatorname{Max}\left(D_{1}, D_{2}\right)$

Stage 2: During stage 2 it is assumed that separation between the core and wrap will not occur, hence the wrap fibre will always remain in the centre with the core wound around it. 
With the wrap fibre now experiencing elongation, it will cause the wrap diameter to decrease, hence the helical radius $\left(r_{2}\right)$ of the core fibre axis will decrease according to equation (A.15), where $R_{2}$ is the radius of the core filament and $d$ is equal to the deformed diameter of the wrap fibre.

$r_{2}=R_{2}+0.5 d$

Like equation (A.5), the definition of Poisson's ratio can allow the deformed diameter of the wrap fibre $(d)$ to be derived as equation (A.16), where $v_{w}$ is the Poisson's ratio of the wrap and $\varepsilon_{w}$ is the axial strain of the wrap fibre.

$d=d_{0}-v_{w} \varepsilon_{w} d_{0}$

During stage 2, the core fibre is wound around the wrap fibre and the critical strain is

exceeded. Figure A.1(e) shows the helical path of the core fibre axis during the second stage. By using Pythagoras's theorem, it is possible to solve for the hypotenuse, by then comparing this against the original length yields equation (A.17) for axial strain $\left(\varepsilon_{c 2}\right)$ in the core during the second stage:

$\varepsilon_{c 2}=\frac{\sqrt{(2 \pi r)^{2}+l_{0}\left(1+\varepsilon_{t}\right)^{2}}-\lambda}{\lambda}$

Equation (A.18) is obtained by comparing Figure A.1(d) and Figure A.1(e):

$l_{0}\left(1+\varepsilon_{w}\right)=\lambda\left(1+\varepsilon_{t}\right)$

(A.18)

This can then be rearranged to give equation (A.19), where $\cos \theta_{0}$ is equal to $\lambda$ divided by $l_{0}$ as found from Figure A.1(a).

$\left(\varepsilon_{w}+1\right)=\cos \theta_{0}\left(1+\varepsilon_{t}\right)$

By substituting equations (A.16), (A.17), (A.18) and (A.19) into equation (A.15), the helical radius of the core fibre axis during the second stage $\left(r_{2}\right)$ can be expressed as by equation (A.20): 
$r_{2}=R_{0}\left(1-v_{c} \frac{\sqrt{4 \pi^{2}\left(R_{0}+\frac{d_{0}}{2}\right)^{2} l_{0}^{2}\left[\cos \theta_{0}\left(1+\varepsilon_{t}\right)\right]^{2}}}{\frac{2 \pi\left(R_{0}+\frac{d_{0}}{2}\right)}{\tan \theta_{0}}}-1\right)+\frac{d_{0}}{2}\left(1-v_{w}\left[\cos \theta_{0}\left(1+\varepsilon_{t}\right)-1\right]\right)$

(A.20)

The effective diameter during the second stage, $D$, is shown by equation (A.21):

$D=2\left(R_{2}+r_{2}\right)$

Substituting equation (A.15) in terms of $R_{2}$ into equation (A.21), before substituting equations (A.16) and subsequently (A.19), the effective diameter is expanded to yield equation (A.22), which varies with the tensile strain $\left(\varepsilon_{t}\right)$ of auxetic yarn:

$D_{2}=4 r_{2}-d_{0}\left(1-v_{w}\left[\cos \theta_{0}\left(1+\varepsilon_{t}\right)-1\right]\right)$

(A.22)

Example analytical calculations: To obtain the Poisson's ratio of the auxetic yarn designed by the wrap filament and the core filament, the initial structure parameters of auxetic yarn (as an example, with polycarbonate as core and nitinol as wrap material, at an axial strain $\varepsilon_{t}$ of 0.16$)$ can be taken as $\left(R_{0}=0.26 \mathrm{~mm}, d_{0}=0.13 \mathrm{~mm}, \theta_{0}=30^{\circ}, v_{c}=0.375, v_{w}=\right.$ $0.3)$.

First the critical strain can be calculated using equation (A.1), where $\theta_{0}$ is in radians: $\varepsilon_{c r i}=$ $\frac{1}{\cos \frac{\pi}{6}}-1=0.155$. Before calculating $r_{2}, l_{o}$ from equation (A.9) must be obtained: $l_{0}=$ $\frac{2 \pi(0.26+0.13 / 2)}{\sin \frac{\pi}{6}}=4.084 \mathrm{~mm}$. Inputting parameters into equation (A.20) for $r_{2}$ yields:

$r_{2}=0.26\left(1-0.375 \frac{\sqrt{4 \pi^{2}\left(0.26+\frac{0.13}{2}\right)^{2} 4.084^{2}\left[\cos \left(\frac{\pi}{6}\right)(1+0.16)\right]^{2}}}{2 \pi(0.26+0.13 / 2) / \tan \left(\frac{\pi}{6}\right)}-1\right)+\frac{0.13}{2}\left(1-0.3\left[\cos \left(\frac{\pi}{6}\right)(1+\right.\right.$ $0.16)-1])=0.296$. Knowing $r_{2}$ enables $D_{2}$ to be evaluated using equation (A.22): $D_{2}=$ $(4 \times 0.464)-0.13\left(1-0.3\left[\cos \left(\frac{\pi}{6}\right)(1+0.16)-1\right]\right)=1.054$. The Poisson's ratio $(v=$ 
$\left.-\frac{\frac{D-2\left(R_{0}+d_{0}\right)}{2\left(R_{0}+d_{0}\right)}}{\varepsilon_{\text {crit }}}\right)$ at the given axial strain may now be expressed as: $v=-\frac{\frac{1.054-2(0.26+0.13)}{2(0.26+0.13)}}{0.155}=$ $-2.26$

\section{Appendix B: Work stored calculations}

Work at breaking point: (assuming a linear profile between origin and yield point, Fig. 7(a))

$W_{B}=F \times d$

$\therefore W_{B 1}=\frac{F \times d}{2}=\frac{250 \times 64 \times 10^{-3}}{2}=8 \mathrm{Nm}$

(A.23a)

$\therefore W_{B 2}=\frac{F \times d}{2}=\frac{250 \times 67 \times 10^{-3}}{2}=8.375 \mathrm{Nm}$

(A.23b)

$\therefore W_{B 3}=\frac{F \times d}{2}=\frac{155 \times 68 \times 10^{-3}}{2}=5.27 \mathrm{Nm}$

(A.23c)

Work at breaking point: (assuming a linear profile between origin and second phase loading,

Fig. 7(a))

$W_{A f t}=F \times d$

$\therefore W_{A f t 1}=\frac{F \times d}{2}=\frac{85 \times 64 \times 10^{-3}}{2}=2.72 \mathrm{Nm}$

(A.24a)

$\therefore W_{A f t 2}=\frac{F \times d}{2}=\frac{85 \times 67 \times 10^{-3}}{2}=2.848 \mathrm{Nm}$

(A.24b)

$\therefore W_{A f t 3}=\frac{F \times d}{2}=\frac{100 \times 76 \times 10^{-3}}{2}=3.8 \mathrm{Nm}$

(A.24c) 
Difference in work:

$W_{D i f}=W_{B}-W_{A f t}$

$\therefore W_{D i f 1}=W_{B 1}-W_{A f t 1}=8-2.77=5.23 N m$

(A.25a)

$\therefore W_{D i f 2}=W_{B 2}-W_{A f t 2}=8.375-2.848=5.527 \mathrm{Nm}$

(A.25b)

$\therefore W_{D i f 3}=W_{B 3}-W_{A f t 3}=5.27-3.8=1.47 N m$

(A. 25c)

Force absorbed by rubber: This force calculation was to show that the difference in load carried before and after the point of failure in Fig. 7(a) was greater than the load carried solely by the nitinol. Breaking Strength $=1 G P a$ (nitinol wire; Flexinol ${ }^{\circledR}$ Muscle Wire ${ }^{\circledR}[26]$, of $0.375 \mathrm{~mm}$ diameter); $F=\sigma A=1 \times 10^{9} \times \frac{\pi\left(0.375 \times 10^{-3}\right)^{2}}{4}=110.45 \mathrm{~N} \quad$ (A.26) 